\title{
77th Annual Meeting of the Swiss Society for Dermatology
} and Venereology

Th. Rufli

Basel

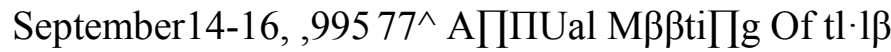

Editor: Prof. Th. Rufli, Basel

Contents

Meleda Disease: Report of Precipitating Factors and Associated

Two Cases Investigated by Diseases in 84 Patients with

Electron Microscopy Granuloma annulare:

E. Frenk, D. Guggisberg, B. Mevorah, A Retrospective Study

D. Hohl (Lausanne) 358 EM. Studer, A.-M. Calza,

J.-H. Saurat (Geneva) 364

Acrodermatitis Enteropathica

Secondary to Crohn's Disease

Livedo-Like Dermatitis

M. Krasovec, E. Frenk (Lausanne) 361 (Nicolau's Syndrome):

A Review of Three Cases

$\mathrm{Ph}$. Ruffieux, D. Salomon,

J.-H. Saurat (Geneva) 368 